\title{
Análise multivariada da esquistossomose no estado de Minas Gerais: análise de componentes principais
}

\author{
Multivariate analysis of schistosomiasis in the \\ state of Minas Gerais: principal component analysis
}

José de Paula Silva1, Salvador Boccaletti Ramos², Monica de Andrade²

\section{RESUMO}

Introdução: A esquistossomose mansônica é uma doença negligenciada no Brasil, logo, relacionada à pobreza e ausência de condições sanitárias. Ocorre na região litorânea do nordeste e se interioriza a partir do estado da Bahia, chegando a Minas Gerais. Objetivo: Agrupar, por meio da análise de componentes principais, as principais variáveis relacionadas à esquistossomose em fatores que possam explicar a variância dos dados. Métodos: Foram avaliadas as notificações de esquistossomose entre os anos de 2007 a 2014, considerando a média por 100 mil habitantes. As variáveis: educação, saúde, economia, infraestrutura e saneamento, população, altimetria e Índice de Desenvolvimento Humano (IDH), foram obtidas no Atlas Brasil; os dados das notificações de esquistossomose foram obtidos no Sistema de Informação de Agravos e Notificação (SINAN). A análise estatística foi realizada a partir da análise de componentes principais, visando identificar os principais fatores que contribuem de forma mais significativa, com a variância dos dados. Resultados: Considerando os critérios propostos, três fatores explicaram mais de $76 \%$ da variância, sendo o primeiro relacionado às variáveis: taxa de analfabetismo em 18 anos ou mais, sem ensino fundamental, mortalidade infantil, extremamente pobre, vulnerável a pobreza, esgotamento sanitário inadequado e população rural. O segundo foi relacionado à variável esgotamento sanitário e o terceiro fator à variável altitude. Conclusão: As variáveis associadas aos fatores, principalmente aquelas pautadas nos determinantes sociais de saúde, demonstram uma forte relação com a ocorrência da esquistossomose. A contribuição do estudo nos remete a possibilidade de agrupar variáveis em fatores que comportam de forma sinérgica e favorecem a incidência da parasitose.

Palavras-chave: esquistossomose; análise fatorial; doenças negligenciadas.

Recebido em: 11/07/2017

Revisado em: 13/01/2018

Aprovado em: 12/03/2018

\begin{abstract}
Introduction: Schistosomiasis mansoni is a neglected disease in Brazil, therefore, related to poverty and lack of sanitary conditions. Occurs in the coastal region of the northeast and spreads inland from the state of Bahia, reaching Minas Gerais. Objective: To group, through component analysis, the main variables related to schistosomiasis in factors that may explain data variance. Methods: Reports of schistosomiasis between the years 2007 to 2014 were evaluated, considering averages per 100 thousand inhabitants. The variables: education, health, economy, infrastructure and sanitation, population, altimetry and Human Development Index (HDI), were obtained from Atlas Brazil; the schistosomiasis notification data were obtained from the injury and notification information system (SINAN). Statistical analysis was based on the analysis of main components, aiming to identify the key factors that contribute most significantly to data variance. Results: Considering the proposed criteria, three factors explained more than $76 \%$ of the variance, the first one related to the following variables: illiteracy rate at 18 years of age or more, no elementary education, child mortality, extremely poor, vulnerable to poverty, inadequate sanitary sewage and rural population. The second was related to the sanitary sewage variable and the third factor to the altitude variable. Conclusion: The variables associated with factors, especially those based on the social determinants of health; show a strong relation with the occurrence of schistosomiasis. The contribution of the study reminds us of the possibility of grouping variables into factors that behave synergistically and favor the incidence of parasitosis.
\end{abstract}

Keywords: schistosomiasis; factor analysis, statistical; neglected diseases.

Autor para correspondência: José de Paula Silva - Universidade do Estado de Minas Gerais - Avenida Juca Stockler 1130 - CEP: $37901-106$ - Bairro Belo Horizonte - Passos (MG) - E-mail: josepaula@gmail.com

Conflito de interesses: nada a declarar 


\section{INTRODUÇÃO}

A esquistossomose mansônica é considerada uma doença negligenciada no Brasil e no mundo, cuja ocorrência está relacionada à pobreza e à ausência de condições sanitárias adequadas ${ }^{1}$.

O Shistosoma mansoni possui um ciclo biológico do tipo heteroxênico, com a presença de um hospedeiro definitivo (homem) e outro intermediário (gastrópodes principalmente do gênero Biomphalaria). No ciclo de vida, os ovos são liberados com as fezes do hospedeiro definitivo, entram em contato com água doce, eclodem, liberam miracídios que infectam os caramujos. Em seguida, desenvolvem os esporocistos e posteriormente cercárias, que são formas aquáticas que nadam e penetram ativamente a pele do hospedeiro definitivo, transformando-se em esquistossômulos, que migram para pulmões e, posteriormente, para o sistema porta-hepático, dando origem às formas adultas ${ }^{2}$.

No Brasil, a esquistossomose está distribuída em uma faixa contínua, ao longo da faixa litorânea da região Nordeste, e a partir do estado da Bahia se interioriza, chegando a Minas Gerais onde segue as principais bacias hidrográficas do estado ${ }^{3}$. Entre as caraterísticas da esquistossomose destaca-se a veiculação hídrica, característica de áreas rurais, além de estar associada às variações ambientais e socioeconômicas ${ }^{4}$.

Estudos ecológicos envolvem um número muito grande de variáveis. Algumas destas podem conter informações mais relevantes cuja escolha como indicador de risco da doença pode ser feita a partir de critérios pouco objetivos. Neste caso, a análise de componentes principais (ACP) permite que, por meio de transformações ortogonais, seja feita a conversão de variáveis em um conjunto que as agrupe em componentes principais.

A análise de componentes principais foi criada por Karl Pearson em $1901^{5}$ e o advento da informática permitiu o seu uso mais intenso, inclusive como ferramenta exploratória em modelos preditivos.

A principal característica da ACP é a redução da dimensão dos dados, técnica que permite verificar padrões de dados cuja expressão sob a forma de fatores pode destacar as semelhanças e diferenças. A partir da determinação dos padrões é possível reduzir as dimensões, agrupando sem perda considerável de informação $0^{6}$.

A escolha das variáveis neste estudo considerou alguns aspectos: em primeiro lugar, os fatores determinantes da ocorrência da esquistossomose, como o fato da parasitose ser de veiculação hídrica e ser considerada uma doença negligenciada, vinculada às condições de pobreza ${ }^{7}$. Em segundo lugar, considerou-se a abordagem da promoção da saúde e a compreensão do conceito de ambientes favoráveis ${ }^{8}$.

Assim, as condições de pobreza e desigualdade aliadas aos fatores ambientais podem nortear a compreensão dos fenômenos que envolvem ou estejam relacionados à incidência de esquistossomose em Minas Gerais.
Este artigo propõe uma análise das variáveis que podem auxiliar a compreensão dos fatores determinantes na ocorrência da esquistossomose mansônica no estado de Minas Gerais, utilizando metodologia de análise multivariada, com o uso da análise de componentes principais.

\section{MÉTODOS}

\section{Tipo e Área de Estudo}

O presente estudo é do tipo ecológico, cuja área envolve o estado de Minas Gerais, que possui uma população estimada de 20.869.101 habitantes, em uma área de $586.520 \mathrm{~km}^{2}$, com 853 municípios. O rendimento mensal domiciliar per capita é de $\mathrm{R} \$ 1.128,00^{9}$.

Segundo dados do Sistema de Informação e Agravos de Notificação (SINAN) do Ministério da Saúde, entre os anos de 2007 a 2014, 482 municípios apresentaram alguma notificação de esquistossomose mansônica, sendo notificados 60.301 casos neste período ${ }^{10}$.

\section{Dados de agravos e secundários}

Os dados secundários que abrangem educação, saúde, socioeconomia, infraestrutura e saneamento, população, Índice de Desenvolvimento Humano foram obtidos no sitio Atlas Brasil, formados pelos dados coletados no recenciamento de 2010 .

O número de casos de esquistossomose em 482 cidades medidos entre 2007 e 2014 foi obtido pelo Sistema de Informação e Agravos de Notificação (SINAN).

Os dados altimétricos dos municípios brasileiros foram obtidos por meio do cadastro de localidades brasileiras do Instituto Brasileiro de Geografia e Estatística (IBGE), cuja publicação fornece os nomes, a subordinação político-administrativa, as coordenadas (latitudes e longitudes) e as altitudes médias das sedes de mais de 21 mil localidades entre municípios, vilas, assentamentos e afins ${ }^{11}$.

Considerando os fatores inerentes ao ciclo de desenvolvimento parasitário do Shistosoma mansoni, bem como os fatores ambientais, socioeconômicos e altimétricos, foram escolhidas as variáveis: taxa de analfabetismo de moradores com 18 anos ou mais (txanalf18), porcentagem de pessoas em domicílios em que ninguém tem fundamental completo (semfund), mortalidade infantil (mort), índice de Gini (Gini), porcentagem de moradores extremamente pobres (extpob), porcentagem de moradores vulneráveis à pobreza (vulpob), porcentagem da população em domicílios com coleta de lixo (com lixo), porcentagem da população em domicílios com energia elétrica (comenerg), porcentagem de pessoas em domicílios com abastecimentos de água inadequados (comaguainad), porcentagem de pessoas em domicílios com paredes inadequadas (comparedeinad), porcentagem de domicílios com rede geral de esgoto ou pluvial (com esgoto), porcentagem de domicílios com esgoto lançado em 
rio, lago ou mar (esgotorio), porcentagem de população rural (\%rural), altitude do município em metros (altit), IDHM geral (IDHM), IDHM educação (IDHM e), IDHM longevidade (IDHM 1), IDHM renda (IDH r) e média de notificações de esquistossomose entre os anos de 2007 a 2014 por 100 mil habitantes (média 2007-2014).

\section{Análise estatística}

A análise estatística foi conduzida em três etapas, utilizando software statistica ${ }^{\circledR}$ da empresa Statsoft. A primeira etapa consistiu na padronização dos dados das variáveis como apresentado:

$\mathrm{Z}=\frac{\mathrm{X}-\mu}{\sigma}$

Sendo

$\mathrm{Z}=$ valor normalizado

$\mathrm{X}=$ valor que se deseja normalizar

$\mu=$ média aritmética da distribuição

$\sigma=$ desvio padrão

A segunda etapa foi a determinação da matriz de correlação linear por meio da seguinte fórmula:

$r=\frac{\sum_{i}\left(x_{i}-\bar{x}\right)\left(y_{i}-\bar{y}\right)}{\sqrt{\sum_{i}\left(x_{i}-\bar{x}\right)^{2} \sum_{i}\left(y_{i}-\bar{y}\right)^{2}}}$

em que:

$\mathrm{x}_{\mathrm{i}}$ é o valor da observação i,

x é a média aritmética da variável $\mathrm{x}$,

$\mathrm{y}_{\mathrm{i}}$ é o valor da variável y e

y é a média da variável y.

Por fim, a última etapa foi a estimação dos componentes principais.

A seleção dos componentes obedeceu a alguns critérios; um deles indicado por Jolliffe ${ }^{12}$ em que se é proposto que a porcentagem acumulada referente a variância total seja superior a $70 \%$.

Os autovalores (eigenvalues) foram determinados a partir de matrizes de covariância ou de correlação. Seu objetivo foi determinar um conjunto de vetores que não são correlacionados e, ao mesmo tempo, independentes, e tenham a capacidade de explicar o máximo da variabilidade dos dados. O critério de Kaiser ${ }^{13}$ sugere que sejam utilizados os fatores superiores a uma unidade, ou autovalor, maior do que um.

\section{RESULTADOS}

Selecionadas as variáveis, estas foram submetidas entre si a testes de correlação. Os resultados podem ser observados na Tabela 1 na qual as colunas e linhas representam as variáveis. Todas as variáveis apresentaram correlações significativas $(p<0,05)$ com alguma outra variável. As correlações não significativas foram assinaladas por sublinhado (Tabela 1).

As medidas de inter-correlação são altamente importantes, pois deve-se ter como critério a existência de correlações que justifiquem a aplicação da análise ${ }^{14}$, sendo que coeficientes de correlação significativos demonstram fortes relações entre variáveis.

Uma vez determinado os coeficientes de correlação, as variáveis que não apresentaram correlação significativa com a média de esquistossomose entre os anos de 2007 a 2014 foram excluídas. Também foram excluídos o índice de GINI e a porcentagem de pessoas em domicílios com moradias de paredes inadequadas, bem como os índices IDHMe, IDHMl e IDHMr, sendo considerado apenas o índice IDHM, visto que este é composto dos demais indicadores de desenvolvimento.

Avaliando as inter-correlações entre as variáveis, a média da incidência da parasitose entre os anos de 2007 a 2014 possui correlação significativa com todas as variáveis nas quais pese que os valores, segundo critérios, indiquem que sejam consideradas correlações leves ${ }^{15}$.

Na Tabela 2 são apresentados os autovalores e a capacidade de explicação (variância total).

Como citado, no critério proposto por Kaiser ${ }^{13}$ devem ser considerado os componentes cujos valores sejam superiores a 1 (um). $\mathrm{Na}$ Tabela 2, os autovalores maiores que um, (fatores 1, 2 e 3) podem explicar $76,60 \%$ da variância dos dados. O número de fatores pode ser confirmado quando utilizado o critério proposto por Cattell ${ }^{16}$ que, baseado em modelo gráfico, considera os componentes anteriores ao ponto de inflexão da curva (Figura 1). Considerando estes dois critérios propostos, podemos observar que os componentes de 1, 2 e 3 explicam mais de $76 \%$ e também correspondem ao ponto de inflexão da curva.

Já na Tabela 3 é possível identificar as ponderações das variáveis, considerando os valores em módulo maiores que 0,7 de maior significância. Assim, é possível verificar que nove variáveis têm maior correlação com o fator 1 , uma variável tem maior correlação com o fator 2 e uma variável tem maior correlação com o fator 3 .

Ainda na Tabela 3 verifica-se que o fator 1 é capaz de explicar $56,20 \%$ da variância. As variáveis: taxa de analfabetismo 18 anos ou mais, sem ensino fundamental, mortalidade infantil, extremamente pobre, vulneráveis à pobreza, esgotamento sanitário inadequado e população rural, foram discriminadas no componente principal; sugerindo que este fator represente um componente denominado 'saúde-social-econômico-ambiental'

Com relação ao fator 2, este contribui com $11,82 \%$ da variância, sendo a principal variável do componente o esgotamento sanitário em rios e lagos. Assim, o fator 2 representa um componente denominado 'saneamento'.

O fator 3 contribui com $8,53 \%$ da variância e seu principal componente é a altitude do município, representando um componente denominado 'altimétrico'. 


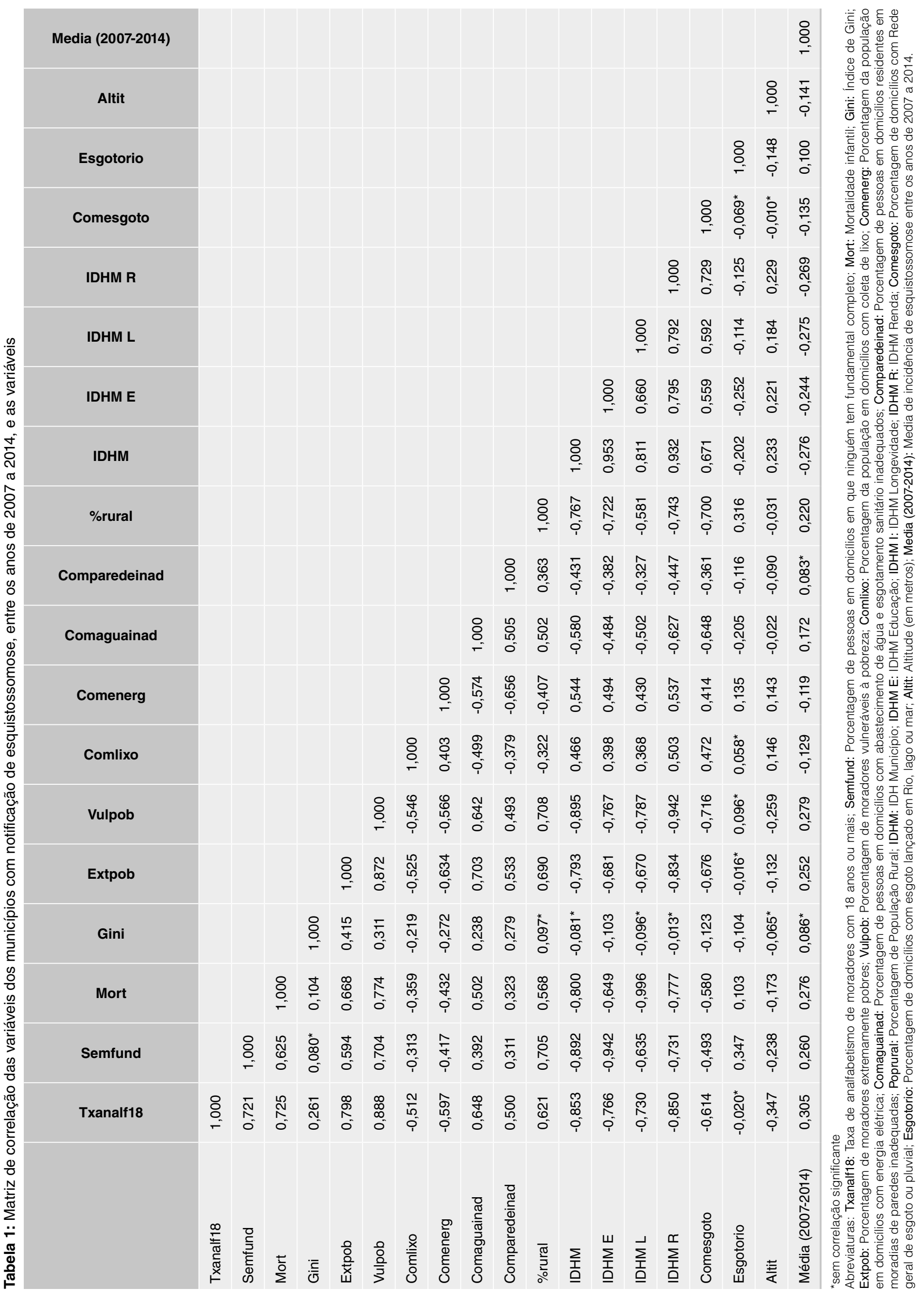


Tabela 2: Autovalores da matriz de correlação e estatística, de acordo com o número de fatores

\begin{tabular}{|c|c|c|c|c|}
\hline Fatores & Autovalor & $\begin{array}{c}\text { Variância } \\
\text { Acumulada }\end{array}$ & $\begin{array}{c}\text { Autovalor } \\
\text { acumulado }\end{array}$ & $\begin{array}{c}\% \\
\text { acumulada }\end{array}$ \\
\hline 1 & 7.306164 & 56.20126 & 7.30616 & 56.2013 \\
\hline 2 & 1.544408 & 11.88006 & 8.85057 & 68.0813 \\
\hline 3 & 1.108580 & 8.52754 & 9.95915 & 76.6089 \\
\hline 4 & 0.691470 & 5.31900 & 10.65062 & 81.9279 \\
\hline 5 & 0.555854 & 4.27580 & 11.20648 & 86.2037 \\
\hline 6 & 0.464249 & 3.57115 & 11.67073 & 89.7748 \\
\hline 7 & 0.358104 & 2.75465 & 12.02883 & 92.5295 \\
\hline 8 & 0.292440 & 2.24954 & 12.32127 & 94.7790 \\
\hline 10 & 0.259630 & 1.99715 & 12.58090 & 96.7762 \\
\hline 11 & 0.186053 & 1.43117 & 12.76695 & 98.2073 \\
\hline 12 & 0.126773 & 0.97518 & 12.89373 & 99.1825 \\
\hline 13 & 0.074349 & 0.57191 & 12.96808 & 99.7544 \\
\hline & 0.031925 & 0.24558 & 13.00000 & 100.0000 \\
\hline
\end{tabular}

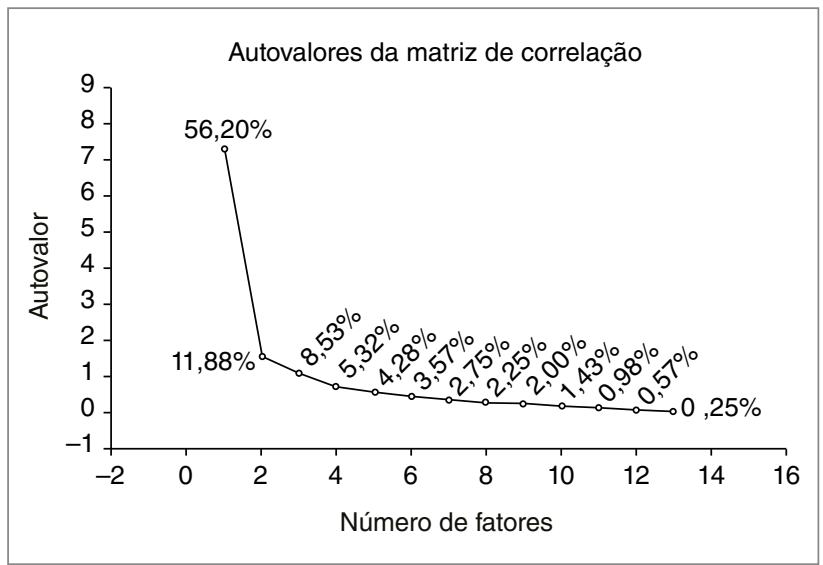

Figura 1: Autovalores da matriz de correlação, valores acumulados representados em gráfico scree.

Tabela 3: Correlação de cada variável avaliada com o respectivo fato

\begin{tabular}{|l|c|c|c|}
\hline Variável $^{*}$ & Fator 1 & Fator 2 & Fator 3 \\
\hline Txanal18 & $-0.908917^{\star}$ & -0.038622 & 0.218001 \\
\hline Semfund & $-0.789606^{\star}$ & 0.413939 & -0.008348 \\
\hline Mort & $-0.802073^{\star}$ & 0.098590 & 0.002362 \\
\hline Extpob & $-0.896268^{\star}$ & -0.159427 & -0.032640 \\
\hline Vulpob & $-0.946338^{\star}$ & 0.018031 & 0.057170 \\
\hline Comlixo & 0.588682 & 0.294888 & -0.148841 \\
\hline Comenerg & 0.659430 & 0.324714 & -0.165136 \\
\hline Comaguainad & $-0.736591^{*}$ & -0.442893 & -0.088397 \\
\hline Poprural & $-0.793283^{\star}$ & 0.252715 & -0.345793 \\
\hline IDHM & $0.942766^{\star}$ & -0.195032 & 0.005966 \\
\hline Comesgoto & $0.779703^{\star}$ & 0.099841 & 0.321799 \\
\hline Esgotorio & -0.106191 & $0.860558^{\star}$ & -0.170154 \\
\hline Altit & 0.230450 & -0.308424 & $-0.864461^{*}$ \\
\hline
\end{tabular}

* Variáveis selecionadas com valor maior que 0,7 (em módulo).

Abreviaturas: Txanalf18: Taxa de analfabetismo de moradores com 18 anos ou mais: Semfund: Porcentagem de pessoas em domicílios em que ninguém tem fundamental completo; Mort: Mortalidade infantil; Extpob: Porcentagem de moradores extremamente pobres; Vulpob: Porcentagem de moradores vulneráveis à pobreza; Comlixo: Porcentagem da população em domicílios com coleta de lixo: Comenerg: Porcentagem da população em domicílios com energia elétrica: Comaguainad: Porcentagem de pessoas em domicílios com abastecimento de água e esgotamento sanitário inadequados; Poprural: Porcentagem de População Rural; IDHM: IDH Município; Comesgoto: Porcentagem de domicílios com Rede geral de esgoto ou pluvial; Esgotorio: Porcentagem de domicílios com esgoto lançado em Rio, lago ou mar; Altit: Altitude (em metros).
A figura 2 apresenta o plano de projeção das variáveis selecionadas (valor maior que 0,7 ) de acordo com os fatores. O sistema permite reduzir a dimensionalidade dos dados projetando em um espaço. No sistema de projeção é possível visualizar os dois principais fatores.

No gráfico bidimensional entre os fatores 1 e 2, a partir do eixo do fator 1, as variáveis: sem ensino fundamental, população rural, mortalidade, vulneráveis a pobreza, taxa de analfabetismo, extremamente pobres e com água inadequada projetam-se à esquerda do eixo, junto a com a média de notificações de esquistossomose/2007-2014. As variáveis: com coleta de lixo, com energia elétrica, com esgoto e IDHm projetam-se à direita (Figura 2).

Ainda nessa figura, o gráfico bidimensional apresenta a principal variável do fator 2 (contribui $11,53 \%$ da variância total) como a 'porcentagem de domicílios que lançam esgoto em rio lago ou mar' e sua correlação com o fator sendo de 0,8605 . Destaca-se que tal correlação é considerada alta e contribui com o entendimento da dinâmica da esquistossomose.

$\mathrm{Na}$ Figura 3, o gráfico bidimensional dos fatores 1 e 3 apresenta a variável altitude sendo projetada em sentido contrário à média de esquistossomose (2007-2014). O fator 3 contribui com 8,53\% da variância e a altitude correlaciona $-0,8644$ com o fator.

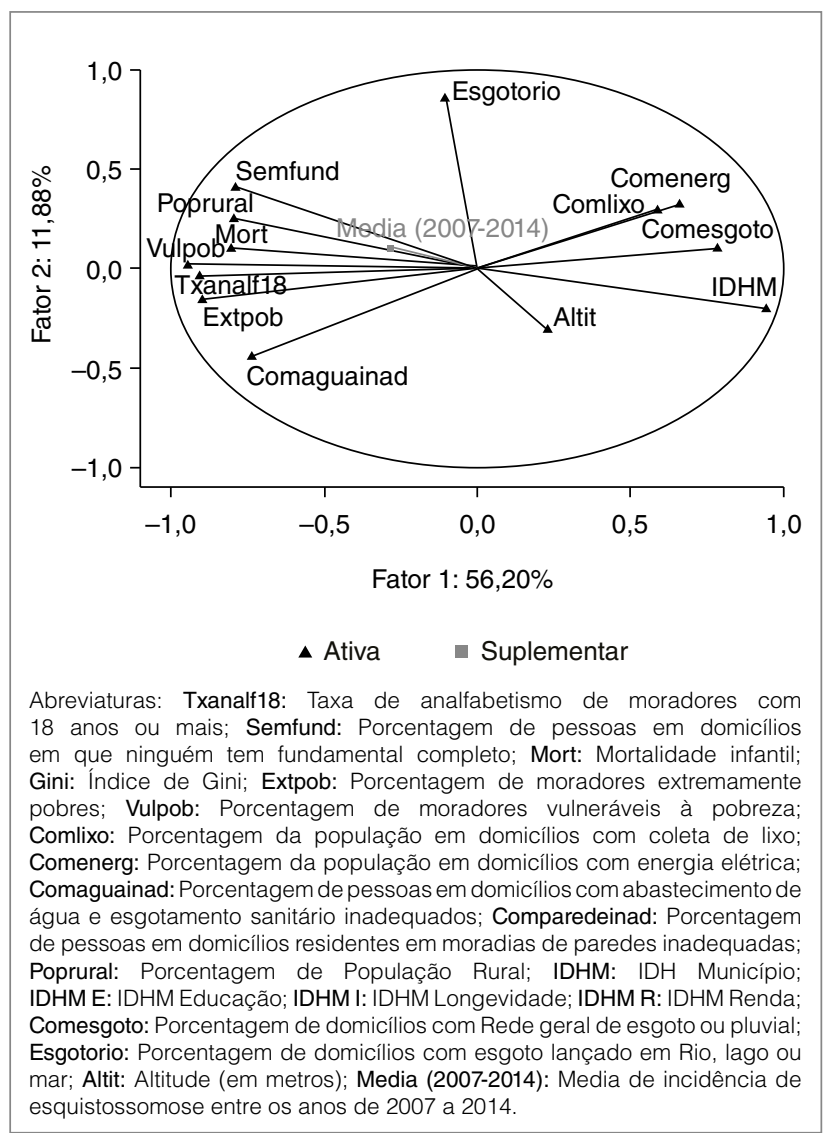

Figura 2: Gráfico bidimensional dos fatores (1 e 2), relacionados com a média de esquistossomose mansônica entre os anos de 2007/2014 no Estado de Minas Gerais. 


\section{DISCUSSÃo}

As três maiores correlações entre a média de incidência de esquistossomose foram analfabetismo, vulnerabilidade à pobreza e mortalidade infantil, cujos resultados parecem corroborar com a compreensão da esquistossomose como uma doença negligenciada. Ela ainda ameaça a saúde de milhões de pessoas no Brasil e no mundo e está relacionada essencialmente à pobreza, enfraquecendo populações, envolvendo os Objetivos de Desenvolvimento do Milênio (ODM). Coincidentemente ou não, a tríade analfabetismo, pobreza e mortalidade é alvo dos ODM, cujos escopos relacionados são erradicar a fome e a pobreza extremas; alcançar a educação primária universal e reduzir a mortalidade infantil ${ }^{17}$.

Considerando o ciclo de desenvolvimento do parasita, o lançamento de esgoto doméstico em água e rio sem sistema de tratamento devem ser considerados como um fator determinante na incidência de esquistossomose.

Mecanismos de controle de transmissão da esquistossomose podem ser realizados por meio do monitoramento de contaminação fecal humana em rios e lagos, incluindo as técnicas de georreferenciamento e identificação genética dos contaminantes fecais, de contagem de unidades formadoras de colônia (determinação quantitativa de coliformes fecais em água), bem como

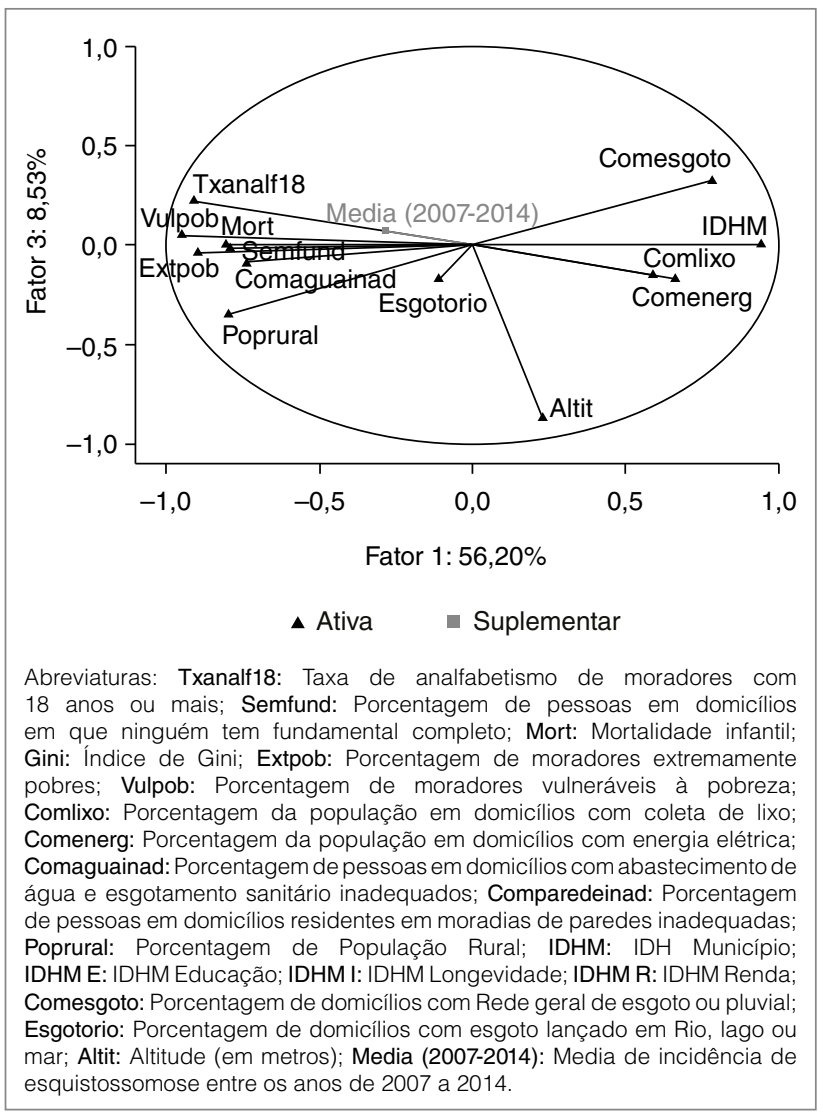

Figura 3: Gráfico bidimensional dos fatores (1 e 3), relacionados com a média de esquistossomose mansônica entre os anos de 2007/2014 no Estado de Minas Gerais. identificação de marcadores genéticos de coliformes fecais humanos em água ${ }^{18}$.

A relação negativa com a altitude coincide com alguns trabalhos, como exemplo, em recente artigo avaliando a esquistossomose em Costa do Marfim (África), utilizando metodologia de distribuição espacial da parasitose identificou-se uma relação negativa entre a altitude e a ocorrência da esquistossomose, sendo aquela a região mais propicia para tal ocorrência, visto que está situada entre 200 e 300 metros de altitude ${ }^{19}$.

Há indicação de uma correlação inversa entre altitude e incidência de esquistossomose, na qual ela pode ser um fator determinante, indicando regiões onde os esforços no combate à doença podem ser inicialmente realizados.

Alguns estudos têm demostrado o risco de transmissão urbana da esquistossomose em função da presença do hospedeiro intermediário, o caramujo do gênero Biomphalaria ${ }^{20}$. O fenômeno foi detectado no estado de Pernambuco onde o êxodo rural levou indivíduos parasitados para regiões urbanas com vetores da parasitose, porém persiste a elevada prevalência em áreas rurais, pois os hábitos socioculturais e condições sanitárias favorecem os processos de transmissão da doença ${ }^{21}$. Em Minas Gerais, a prevalência da parasitose na área rural ainda é significativa, pois apesar da população rural deste estado ser de $14,7 \%$ no período avaliado, $42,01 \%$ das notificações de esquistossomose eram de residentes da área rural ${ }^{22}$.

Ambientes desfavoráveis, com esgotamento sanitário, energia elétrica, coleta de lixo e o IDH são antagônicos à esquistossomose e podem ser agrupados em único fator. Por outro lado, taxa de analfabetismo, pobreza, ausência de água encanada e esgoto podem ser agrupadas também em um único fator e acompanham a parasitose. Esses fatores já foram relacionados à prevalência da doença em Minas Gerais, em modelos de regressão linear múltipla cujo escopo identificou condições que favorecem a disseminação da doença, entre os quais pode-se citar topografia, saneamento e desenvolvimento humano ${ }^{23}$.

Destaca-se a preocupação com os fatores ambientais e saneamento identificados neste estudo, que sofrem com a inépcia de órgãos ambientais e gestores municipais, onde muitos municípios mineiros sofrem com deficiências de coletas de lixo, ausência de esgoto e fornecimento de água inadequada ${ }^{24}$.

Conclui-se que, ao longo dos anos, medidas preventivas e profiláticas não têm demonstrado o resultado desejado, haja vista a série história de notificações de esquistossomose em Minas Gerais. Espera-se que abordagens que envolvam a criação de ambientes favoráveis possam ser fundamentais na erradicação da doença, contribuindo para inibição da veiculação hídrica da parasitose.

As variáveis associadas aos fatores, principalmente aquelas pautadas nos determinantes sociais de saúde, demonstram uma forte relação com a ocorrência da esquistossomose. A contribuição do estudo nos remete a possibilidade de agrupar variáveis em fatores, que comportam de forma sinérgica, e favorecem a incidência da parasitose. 


\section{REFERÊNCIAS}

1. Lindoso JAL, Lindoso AABP. Neglected tropical diseases in Brazil. Rev Inst Med Trop. 2009; 51(5):247-53. http://dx.doi.org/10.1590/S0036-46652009000500003

2. Carvalho OS, Coelho PMZ, Lenzi HL. Schistosoma mansoni \& Esquistossomose: uma visão multidisciplinar. FIOCRUZ, 2008.

3. Brasil. Ministério da Saúde. Vigilância da Esquistossomose Mansoni: diretrizes técnicas. 4ed. Brasília: Ministério da Saúde, 2014

4. Saucha CVV, Silva JAM, Amorim LB. Condições de saneamento básico em áreas hiperendêmicas para esquistossomose no estado de Pernambuco em 2012. Epidemiol Serv Saúde. 2015;24(3):497-506.

http://dx.doi.org/10.5123/S1679-49742015000300015

5. Person FRSK. On lines and planes of closest fit to system of points in space. Phil Mag J Sci. 1901;2(11):559-72. http://dx.doi.org/10.1080/14786440109462720

6. James G, Witten D, Hastie T, Tibshirani R. An introduction to statistical learning: with Applications in R. London: Springer; 2013.

7. Departamento de Ciência e Tecnologia, Secretaria de Ciência, Tecnologia e Insumos Estratégicos, Ministério da Saúde. Doenças negligenciadas: estratégias do Ministério da Saúde. Rev Saúde Pública. 2010;44(1):200-2 http://dx.doi.org/10.1590/S0034-89102010000100023

8. Sundsvall DD. Promoção da saúde e ambientes favoráveis à saúde. III Conferência Internacional de promoção da saúde. Disponível em: http://bvsms.saude.gov.br/bvs/publicacoes/ declaracao_sundsvall.pdf. Acesso em: 12 jul. 2017.

9. Instituto Brasileiro de Geografia e Estatística (IBGE). Minas Gerais. Disponível em: http://www.ibge.gov.br/estadosat/perfil. php?sigla=mg. Acesso em: 12 jul. 2017

10. Sistema de Informação de Agravos de Notificação (SIAN). Doenças e agravos. Disponível em: http://portalsinan.saude.gov.br/. Acesso em: 12 jul. 2017.

11. Instituto Brasileiro de Geografia e Estatística (IBGE). Cadastro nacional de localidades. Disponível em: http://geoftp.ibge.gov.br/ Organizacao/Localidades. Acesso em: 12 jul. 2017.

12. Jolliffe IT. Principal component analysis. 2nd ed. New York: Springer; 2002.

13. Kaiser HF. The application of electronic computers to factor analysis. Educat Psychol Measur. 1960;20(1):141-51.
14. Hair Jr JF, Black WC, Babin BJ, Anderson RE, Tatham RL. Análise multivariada de dados. Porto Alegre: Bookman, 2009.

15. Ladis JR, Koch GG. The measurement of observer agreement for categorical data. Biometrics. 1977;33(1):159-74. http://dx.doi.org/10.2307/2529310

16. World Health Organization (WHO). Working to overcome the global impact of neglected tropical diseases: first WHO report on neglected tropical diseases. Geneva: WHO, 2010.

17. Cattell RB. The Scree Test for the number of factors. Multivariate Behav Res. 1966;1(2):245-76. http://dx.doi.org/10.1207/s15327906mbr0102_10

18. Ponce-Terashima R, Koskey AM, Reis MG, McLellan SL, Blanton RE. Sources and distribution of surface water fecal contamination and prevalence of schistosomiasis in a Brazilian village. PLoS Negl Trop Dis. 2014;8(10):e3186. https://doi.org/10.1371/journal.pntd.0003186

19. Assaré RK, Lai YS, Yapi A, Tian-Bi YN, Ouattara M, Yao PK, et al The spatial distribution of Schistosoma mansoni infection in four regions of western Côte d'Ivoire. Geospat Health. 2015;10(1):345. https://dx.doi.org/10.4081/gh.2015.345

20. Gomes ECS, Mesquita MCS, Rehn VNC, Nascimento WRC, Loyo R, Barbosa CS. Transmissão urbana da esquistossomose: novo cenário epidemiológico na Zona da Mata de Pernambuco. Rev Bras Epidemiol. 2016:19(4):822-34. http://dx.doi.org/10.1590/1980-5497201600040012

21. Barreto MS, Gomes ECS, Barbosa CS. Turismo de risco em áreas vulneráveis para a transmissão da esquistossomose mansônica no Brasil. Cad Saúde Pública. 2016;32(3):e00190815. http://dx.doi.org/10.1590/0102-311X00190815

22. Silva JP, Andrade M. Perfil epidemiológico da esquistossomose mansônica em Minas Gerais. Investigação. 2016;15(6).

23. Fonseca F, Freitas C, Dutra L, Guimarães R, Carvalho O. Spatial modeling of the schistosomiasis mansoni in Minas Gerais State, Brazil using spatial regression. Acta Trop. 2014;133:56-63. http://dx.doi.org/10.1016/j.actatropica.2014.01.015

24. Anjos MP, Leonardi FA, Tavares Júnior JB, Ferreira L. Planejamento urbano: o mapeamento do saneamento básico na margem esquerda do Ribeirão Pitanga ou Furnas, nos municípios de Bueno Brandão e Ouro Fino, Minas Gerais-Brasil. SBGFA Ferramenta Leitura. 2017;1:133-45. https://doi.org/10.20396/sbgfa.v1i2017.1784 